\title{
Imaging the elite golfer
}

\author{
P. J. O'Connor • R. Hawkes
}

Published online: 7 December 2012

(C) ISS 2012

Imaging in professional golf is, as in many sports, becoming increasingly important. The value of magnetic resonance (MR) first came to the attention of golfers in 1974 with the MR diagnosis of a hook of hamate stress fracture. This came after a 7-year struggle with this injury for John Cook, one of the US tours top professionals. The events are detailed graphically in John Feinstein's “A good walk spoiled", which highlights the difficulties professional golfers found in gaining consistent reliable medical opinions. This results from the unusual injury patterns in golf, the nomadic nature of the professional golfer's life, the increasing length of the tours, and the huge geographic spread of the venues.

Fortunately, the structure of the Professional Golfers' Association Tour in the USA and the European Tour lends itself to the development of coordinated player medical services. The players are essentially the shareholders in the tours and have a great influence on how the tour uses its funds to support the game. Obviously, prize money remains the top priority, but consistent high-quality medical services are now viewed by the players as vital.

The last 10-15 years have seen professional golfers becoming busier both on and off the course with less time spent in any one place. As a result, medical care is difficult to access and varied. Tournament medical services are usually satisfactory, but provide little continuity and aim merely

P. J. O'Connor $(\bowtie)$

Leeds Teaching Hospitals trust,

Leeds, UK

e-mail: philip.o'connor@leedsth.nhs.uk

R. Hawkes

European Tour, Wentworth Drive,

Virginia Water,

Surrey G025 4LX, UK to provide in-play support rather than provide a more complete management plan.

Consequently, in Europe, a more comprehensive sports medicine service is supported by the tour. This is provided by four sports medics who complement the existing tournament physiotherapist services. Medical cover is provided on the 2 days before events when players are practising and is separate from the tournament doctor service. The service is mobile, using a purposely built extendable medical and training unit that moves between tournaments and includes physiotherapy, gym, consultation rooms, an imaging review workstation, and diagnostic and interventional ultrasound.

As in other areas of sports medicine, radiology is vital to the delivery of these services for diagnosis and intervention. By using electronic transfer, the sports medicine team is able to send magnetic resonance imaging (MRI) studies performed locally to anywhere in the world, to a supporting radiologist who reports on them almost immediately and liaises with the referrer. Regular visits to events by the radiologists are clinically invaluable, offering a case review, and diagnostic and interventional services. It also improves team working, and provides an opportunity for teaching and research. The incorporation of all these services provides a comprehensive central base for the players' medical data combining clinical assessment, physiotherapy, and imaging in a truly multidisciplinary setting. This concentration of medical data and skills has led to a much greater understanding of the patterns of injury seen in professional golf.

Injury patterns differ between professional and amateur golfers, mainly as a result of swing mechanics and the increased number of repetitions [1]. The professional golf swing is much more consistent, channeling forces through the same areas of the musculoskeletal system on a repeated basis. This and the increased levels of activity result in a high incidence of stress injury to the kinetic chain and spine. 
Amateur golfers are, on average, older, play less, and encounter more random acute traumatic or exacerbations of pre-existing conditions than overuse injuries.

In addition, and, as with most sports, golf has benefited from a combination of better equipment and increasing physicality, which has resulted in longer driving distances (of the golf ball!), which is now a key feature of the modern game. There has been a trend in the golf swing to turn the shoulders away from the ball and leave the hips behind in what is thought to coil the spine like a spring and is referred to as the $\mathrm{X}$ factor [2]. This has led to a tendency to create an adverse amount of torque and compression in the spine, with a resulting increase in injuries seen in this area. It is important that the radiologists understand the mechanics of the golf swing and understand the importance of the abnormalities present. Any areas of decreased spinal mobility, either congenital or resulting from disc disease, can have a dramatic effect in the areas of the spine to limb girdle attempting to compensate for the areas of decreased movement. For example, a player with reduced mobility in the thoracic spine can compensate with increased shoulder movement and presents with shoulder problems or even sternoclavicular disease. Clinical correlation is vital in golfers, and structuring medical services to provide a multidisciplinary approach is essential to developing services.

The leading wrist is the most commonly injured area in professional golf [1]. The leading wrist undergoes complex swing mechanics moving from ulnar to radial deviation and then back to ulnar deviation during the swing. There is also movement from pronation, to supination and flexion to extension through impact. This impact is substantial as, with irons, the club face hits the ground taking a divot in order for the player to impart spin, vital in controlling shots.

Injuries result from both the forces channeled through the wrist either from the repetition of these movements or the club shaft. Movement-related conditions commonly seen in the wrist include extensor carpi ulnaris tenosynovitis or instability. Osseous impaction syndromes are frequently seen in the lead wrist. Dorsal rim impingement/impaction [3-5] is common and relatively easy to diagnose both clinically and from an imaging viewpoint. Imaging is of value in showing carpal bosses, dorsal intra-articular bodies, and dorsal ganglia, which can result in players being more susceptible to dorsal impaction. Triquetrohamate impaction is common, though difficult to diagnose with imaging until there is established cartilage loss.

The injury patterns from the club shaft vary with the player's grip and the length of the shaft. We have seen bone stress reactions and stress fractures in the hamate, pisiform, and scaphoid.

On the radial aspect of the wrist, DeQuervain's tenosynovitis is the most commonly encountered condition $[1,6]$. Occasionally, players will strike an object with the club unexpectedly and this can produce an acute wrist injury. In this situation, intrinsic ligament injury and extensor carpi ulnaris subsheath $[7,8]$ disruption are most common. Often, these acute conditions require surgical repair to allow the player to return to play at pre-injury levels.

Lead shoulder-related symptoms are also relatively common. In the lead shoulder, golfers encounter a combination of anterior capsular laxity and posterior capsular contraction, which can lead to an abnormal range of movements and secondary impingement. The mechanism behind this is thought to result from the follow-through and is similar to the development of glenohumeral internal rotation deficit (GIRD) in throwing athletes [9].

In the lead hip, golfers encounter labral injury and secondary osteoarthritis.

Problem patients are reviewed by a therapist, sports physician, coach, and other interested parties (nowadays this can frequently include management teams). Diagnostic and therapeutic ultrasound is performed on site and local MRI facilities are identified before each event. The European tour has pre-defined MR protocols available to players who are advised to have these protocols used when having an MR scan undertaken away from tour events.

This imaging service is now in its fifth year and represents what we feel is only the beginning of structured medical services for this sport. Even in the short time it has been running, the consistent multidisciplinary team approach is greatly advantageous to both tour players and medical teams, with increased knowledge levels regarding golf injuries and improved diagnosis and appropriate intervention throughout our medical specialties. The European tour is thriving, having produced the current top three world-ranked golfers. This has been achieved at a relatively modest cost to the tour of $£ 5,000$ per year, in addition to the physiotherapy and sports medicine service.

Clearly, there would be benefit to bringing this kind of structured approach to medical services in other sports. Whether this is possible is open to debate; the structure of the European Tour with strong player influence has been a major factor in the development of services. It has not been emulated in the USA because of the tight state licensing system, which, ironically, makes it more difficult to cross borders and provide the same continuity enjoyed in Europe.

Professional tennis would seem the most similar lifestyle sport to golf, though the development of this type of service requires strong player support and a degree of vision. We strongly recommend becoming involved in delivering these kinds of structured medical services. From a professional viewpoint, it is exceptionally rewarding clinically to have a greater understanding of the sport and feel in someway part of the organisation of a sport.

Acknowledgements Our thanks to the European Tour Medical team: Dr. Jane Dunbar; Dr. Tim Swann; Mr. Rob Hillman and his European 
Tour Physiotherapy Team; Dr. Rob Campbell, Dr. Philip Robinson, Mr. Doug Campbell and members of the European Tour Upper Limb and Radiology Advisory Board.

\section{References}

1. McCarroll J. Overuse injuries of the upper extremity in golf. Clin Sports Med. 2001;20(3):469-79.

2. McLean Jim. Widen the Gap. Golf Magazine; December 1992.

3. Linscheid RL, Dobyns JH. Athletic injuries of the wrist. Clin Orthop Relat Res. 1985;198:141-51.
4. Gabel GT. Gymnastic wrist injuries. Clin Sports Med. 1998;17:61121.

5. Rettig AC. Athletic injuries of the wrist and hand: part II: overuse injuries of the wrist and traumatic injuries of the hand. Am J Sports Med. 2004;32:262-73.

6. Theriault G, Lachance P. Golf injuries. An overview. Sports Med. 1998;26:43-57.

7. Eckhardt WA, Palmer AK. Recurrent dislocation of extensor carpi ulnaris tendon. J Hand Surg (Am). 1981;6(6):629-31.

8. Osterman AL, Moskow L, Low DW. Soft tissue injuries of the hand and wrist in racquet sports. Clin Sports Med. 1988;7:329-48.

9. Hovis WD, Dean MT, et al. Posterior instability of the shoulder with secondary impingement in elite golfers. Am J Sports Med. 2002;30 (6):886-90. 